\title{
Lattice Boltzmann modelling Knudsen layer effect in non-equilibrium flows
}

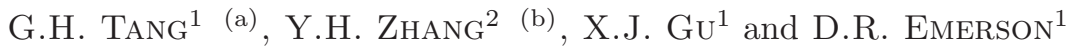 \\ 1 Computational Science and Engineering Department, Daresbury Laboratory, Warrington, \\ WA4 $4 A D, U K$ \\ 2 Department of Mechanical Engineering, University of Strathclyde, Glasgow, G1 1 XJ, UK
}

PACS 05.10.-a - Computational methods in statistical physics and nonlinear dynamics

PACS 47.45.-n - Rarefied gas dynamics

PACS $47.11 .-j$ - Computational techniques in fluid dynamics

\begin{abstract}
Due to its intrinsically kinetic nature, lattice Boltzmann (LB) approach to simulating non-equilibrium gas flows has recently attracted significant research interest. Compared with other kinetic methods, it can offer a significantly smaller computational cost. To capture the nonlinear high-order rarefaction phenomena in gas flows, a geometry-dependent gas local mean free path has been proposed to be implemented in our "high-order" LB model. A series of tests on rarefaction effects and the Knudsen layer interference have been carried out and the simulation results demonstrate our LB model's capability for highly non-equilibrium flows.
\end{abstract}

Introduction. - Understanding fluid flows possessing substantial non-equilibrium effects poses a long-standing challenge to fundamental statistical physics as well as many other science and engineering disciplines [1]. With rapid development of micro/nano fabrication technology, gas flows in micro/nano-fluidic devices have found a broad range of applications. Flows in these miniaturised devices are often non-equilibrium, which can be characterised by the Knudsen number (Kn), defined as the ratio of the gas molecular mean free path $\lambda$ to the device characteristic length $L$. The Knudsen layer is the local non-equilibrium region extending several mean free paths from the wall. The momentum and heat fluxes, which are of most interest to designers in microflow and nanoflow applications, usually transfer from the boundaries to the bulk flow through this Knudsen layer. Because flows in the Knudsen layer are not in local thermodynamic quasi-equilibrium, the linear constitutive relations for shear stress and heat flux, which are assumed in the Navier-Stokes (NS) equations, are no longer valid in the Knudsen layer [2]. Figure 1 shows the schematic diagram of the velocity structure in the Knudsen layer of a shear driven flow. Although the NS equations with macro slip boundary condition can accurately predict the flow field outside the Knudsen layer, they fail to capture the flow characteristics in the Knudsen layer [3].

To simulate highly non-equilibrium flows, whether a model can capture the Knudsen layer flow characters becomes essential because the Knudsen layer forms a large proportion of the flow domain. Traditionally, kinetic methods such as directly solving the Boltzmann equation or the direct simulation Monte Carlo (DSMC) method can offer accurate descrip-

\footnotetext{
(a) Electronic mail: g.tang@dl.ac.uk

(b) Electronic mail: yonghao.zhang@strath.ac.uk
} 
tion of non-equilibrium flows. However, these methods are computationally impractical for micro/nano-fluidic system simulation [4]. Significant effort has been made to develop computationally efficient and numerically accurate methods, e.g. extended hydrodynamic methods [5,6], hybrid model [7], the information preservation method for DSMC [8], and reducing the statistical scatter associated with Monte Carlo methods $[9,10]$. Recently, the lattice Boltzmann (LB) methodology has also been attempted to model non-equilibrium gas flows [4,11-28]. One important advantage of the LB methodology is that it is kinetic in nature, so does not suffer the closure and boundary condition problems associated with high-order continuum methods such as Grad's 13-moment model.

To accurately describe non-equilibrium gas dynamics beyond the NS level, high-order LB models have been proposed [21,29]. Ansumali et al. [31] have demonstrated that the highorder LB models can enhance performance and capture nonlinear rarefaction phenomena away from wall boundary. However, it appears not being able to predict flow behavior in the Knudsen layer which may be due to lack of appropriate wall boundary conditions (see figure 6 for more detailed comparison with the results of the linearised Boltzmann equation). The other high-order models are also not sufficiently accurate to describe Knudsen layer flow behavior [33]. Unfortunately, the roots of Hermite polynomials of the high-order are irrational, so that the discrete velocities cannot match lattice nodes. Additional effort, such as point-wise interpolation [32], is required. It dramatically increases the computational cost because it essentially becomes an off-lattice discrete velocity method for solving the kinetic Boltzmann equation. Recently, Chikatamarla and Karlin [29] have reported to resolve this issue of fitting Hermite polynomials' solution on lattice.

Efforts have also been made to capture flow characteristics in the Knudsen layer. For example, the regularisation methods add an additional term which considers the omitted high-order moments effect $[24,27,34]$, which are in the same spirit of the regularised moment methods [5,35]. Similarly, Szalmás [28] recently proposed a mechanism to add high-order moments effect through a fast relaxation model as proposed by Gorban [36]. We have also considered the wall effect on gas mean free path in the standard lattice BathnagarGross-Krook (BGK) model [22,26] and our simplified "high-order" D2Q13 model [33] which was inspired by the concept of rational number approximation [29]. However, our previous treatment of the wall effect on the mean free path is difficult to deal with complex geometries and the Knudsen layer overlap effect. In this paper, we will improve our "high-order" LB model capability for highly non-equilibrium flows by incorporating a geometry-dependent local mean free path, an idea appeared in the work of Stops [37] and Guo et al. [38]. The concept of local mean free path is consistent with the mesoscopic nature of LB methodology, an analogous notion was also used by Chen et al. [39] for the simulation of turbulence flows.

Local mean free path. - The mean free path concept, which describes the average distance a gas molecule travels between consecutive collisions, is central to modelling the transport phenomena in gases. When the mean free path $\lambda$ becomes comparable with the linear dimension $L$ of a finite system, the wall boundaries will affect the local mean free path. Usually, the mean free path is determined via the relation to the macroscopic properties such as viscosity, pressure and temperature, e.g. $\lambda=\frac{\mu}{p} \sqrt{\frac{\pi R T}{2}}$, where $\mu$ is the viscosity, $p$ is the gas pressure, $R$ is the gas constant, and $T$ is temperature. However, the presence of wall will reduce the local mean free path at the near wall region. For highly non-equilibrium flows, collisions with the wall rather than intermolecule collisions dominate the flow characteristics.

The wall effect on reducing the local mean free path has been discussed in our previous work, which was largely based on semi-empirical data obtained by other numerical simulations including DSMC method. Although significant improvement of LB model performance has been demonstrated, our previous approach has difficulties in treating complex geometries and Knudsen layer interference. Here, we will adopt the approach used by $[37,38]$ to re-evaluate the wall effect on the local mean free path. For an unbounded ideal gas, we assume that the probability of a gas molecule can travel between two consecutive collisions 


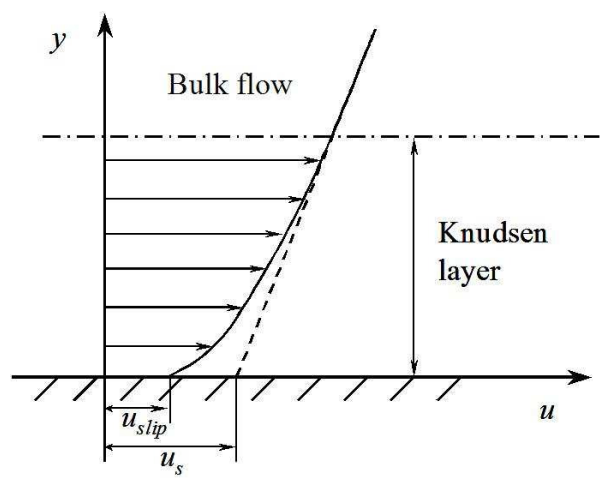

Fig. 1: Schematic diagram showing the actual microscopic slip $\left(u_{\text {slip }}\right)$ and the predicted macroscopic slip $\left(u_{s}\right)$ based on Navier-Stokes equation with macro slip boundary condition within the Knudsen layer for Kramers' problem.

with other gas molecules at location $\mathbf{x}$ and $\mathbf{x}+\mathbf{d x}$ is $P(\mathbf{x})$. This probability function has been asserted in the kinetic theory $[37,38]$ as

$$
P(\mathbf{x})=\exp \left(\frac{-\mathbf{x}}{\lambda_{0}}\right) \mathbf{d}\left(\frac{\mathbf{x}}{\lambda_{0}}\right) .
$$

In the wall region, the gas molecular may collide with wall before hitting another gas molecule, so that local mean free path is smaller than the bulk value $\lambda_{0}$. The wall effect on local mean free path for any given geometry can be derived from the above general Eq.(1).

If we consider an ideal gas bounded by two parallel plates at $y=0$ and $y=L$, the local mean free path of the molecules at a distance $y(0<y<L)$ from the lower plate can be calculated by $[37,38]$

$$
\lambda(y)=\lambda_{0}\left[1+(\gamma-1) \exp (-\gamma)-\gamma^{2} \int_{\gamma}^{\infty} t^{-1} \exp (-t) d t\right],
$$

where $\gamma=y / \lambda_{0}$ for those molecules moving toward $y=0$ and $\gamma=(L-y) / \lambda_{0}$ for those moving toward $y=L$. Since a molecule can move toward these two walls with the same probability, the local mean free path for all the molecules in the flow domain can be determined by averaging these two parts. For $y=0$ or $y=L$ at the wall, we have $\gamma=L / \lambda_{0}$.

Figure 2 presents the local mean free path profiles between two parallel plates for $K=$ $0.01-10$, where $K$ is the rescaled Knudsen number, i.e. $K=(\sqrt{\pi} / 2) K n$. It is apparent that the local mean free path near the walls are significantly reduced. The local mean free path at the wall is only half of the bulk value when the Knudsen number is small. However, with increasing Knudsen number which means the Knudsen layers start to overlap, the local mean free path at the wall decreases further. For example, when $K=0.3$, the local mean free path at the wall is about 0.49 rather than 0.5 . In addition, the wall effect is also felt in the centre of the two plates so that the local mean free path in the center is smaller than the bulk value. For example, when $K=0.2$ and $K=0.3$, the local mean free path at the centre is about $0.95 \lambda_{0}$, and $0.88 \lambda_{0}$, respectively, rather than $\lambda_{0}$.

If we remove the upper plate so that gas flow is only affected by the lower plate at $y=0$, the local mean free path can then be determined as $[37,38]$

$$
\lambda(y)=\frac{\lambda_{0}}{2}\left[2+(\gamma-1) \exp (-\gamma)-\gamma^{2} \int_{\gamma}^{\infty} t^{-1} \exp (-t) d t\right],
$$

where $\gamma=y / \lambda_{0}$. Figure 3 shows that the local mean free path at the wall is half of the bulk value. Moreover, we can see that the thickness of Knudsen layer is $2.33 \lambda_{0}$ if we define its value at $\lambda(y) / \lambda_{0}=0.98$. 


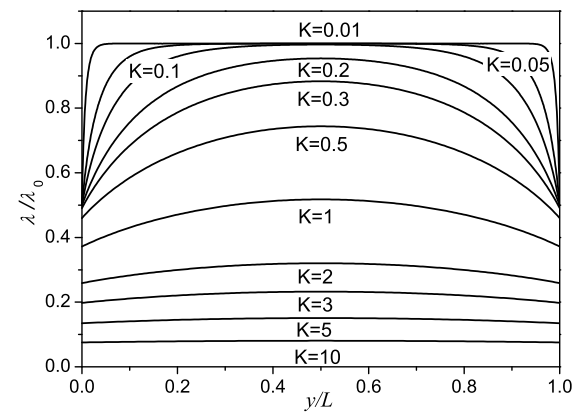

Fig. 2: The local mean free path profiles between two plates.

Lattice BGK model. - The details about the lattice BGK model are widely available e.g. $[40-42]$

$$
\frac{\partial f_{k}}{\partial t}+e_{k i} \frac{\partial f_{k}}{\partial x_{i}}=-\frac{f_{k}-f_{k}^{e q}}{\phi}+\frac{\left(e_{k i}-u_{i}\right) F_{i}}{c_{s}^{2} \rho} f_{k}^{e q},
$$

where $f_{k}$ is the velocity distribution function, $f_{k}^{e q}$ is the distribution function at equilibrium, $e_{k i}$ is the lattice velocity, $u_{i}$ is the macroscopic velocity, $F_{i}$ is the external force, $c_{s}$ is the sound speed of the lattice fluid, $\rho$ is the density, and $\phi$ is the relaxation time. After discretizing Eq. (4), we obtain

$$
f_{k}\left(\mathbf{x}+\mathbf{e}_{k} \delta t, t+\delta t\right)-f_{k}(\mathbf{x}, t)=-\frac{1}{\tau}\left[f_{k}(\mathbf{x}, t)-f_{k}^{e q}(\mathbf{x}, t)\right]+\delta t \frac{\left(e_{k i}-u_{i}\right) F_{i}}{c_{s}^{2} \rho} f_{k}^{e q}(\mathbf{x}, t),
$$

where $\tau=\phi / \delta t$ is the nondimensional relaxation time and $\delta t$ is the time step.

For a two dimensional, thirteen-velocity lattice model (D2Q13) [33], the equilibrium distribution function can be expressed as:

$$
\begin{array}{r}
f_{k}^{e q}=\rho \omega_{k}\left[1+\frac{e_{k i} u_{i}}{c_{s}^{2}}+\frac{\left(e_{k i} u_{i}\right)^{2}}{2 c_{s}^{4}}-\frac{u_{i} u_{i}}{2 c_{s}^{2}}+\frac{\left(e_{k i} u_{i}\right)^{3}}{2 c_{s}^{6}}-\frac{3\left(e_{k i} u_{i}\right)\left(u_{i} u_{i}\right)}{2 c_{s}^{4}}\right], \\
\omega_{0}=\frac{3}{8} ; \quad \omega_{k}=\frac{1}{12}, k=1-4 ; \quad \omega_{k}=\frac{1}{16}, k=5-8 ; \quad \omega_{k}=\frac{1}{96}, k=9-12,
\end{array}
$$

where $c_{s}^{2}=c^{2} / 2$ and $c=\sqrt{2 R T}$. The lattice velocities, $e_{k}$, are given by $e_{0}=0 ; e_{k}=$ $\left[\cos ((k-1) \pi / 2, \sin ((k-1) \pi / 2)] c\right.$ for $k=1-4 ; e_{k}=[\cos ((k-5) \pi / 2+\pi / 4)$,

$\sin ((k-5) \pi / 2+\pi / 4)] \sqrt{2} c$ for $k=5-8 ; e_{k}=[\cos ((k-1) \pi / 2), \sin ((k-1) \pi / 2)] 2 c$ for $k=9-12$.

Similar to our previous work [4,17], the Knudsen number in a D2Q13 lattice BGK model can be related to the relaxation time and the local mean free path:

$$
\tau=\left(\frac{\lambda}{\lambda_{0}}\right) \sqrt{\frac{\pi}{8}}\left(\frac{c}{c_{s}}\right) K n_{0} N_{L}+\frac{1}{2},
$$

where $N_{L}=L / \delta y$ is the number of lattice in characteristic length direction, $\delta y$ is the lattice length, and $K n_{0}$ is the bulk Knudsen number based on the bulk mean free path $\lambda_{0}$ and the characteristic length $L$. Clearly, the dimensionless relaxation time $\tau$ is dependent on the boundary geometry and the specific locations.

Many boundary conditions have been developed to capture velocity slip and temperature jump at the wall, e.g. bounce back, specular reflection, a combination of the above two [30], kinetic boundary condition [43], and a virtual-wall collision scheme [19]. Here, 


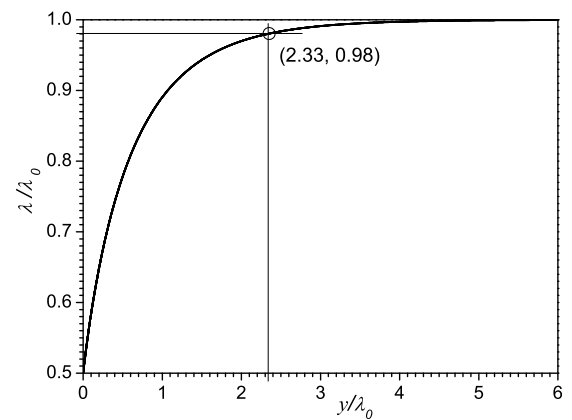

Fig. 3: The local mean free path profile in a half space Kramers' problem where $y$ is the distance from the plate.

the Maxwellian kinetic boundary condition considering tangential momentum accommodation coefficient is applied $[17,43]$. The unknown reflected distribution function $f_{k}$ can be determined by the incident $f_{k^{\prime}}$ :

$$
\begin{aligned}
f_{k}(\mathbf{x}, t+\delta t)= & (1-\alpha) f_{k^{\prime}}(\mathbf{x}, t+\delta t)\left|\left(\mathbf{e}_{k^{\prime}}-\mathbf{u}_{w}\right) \cdot \mathbf{n}\right| \\
+\alpha & \frac{\sum_{\left(\mathbf{e}_{i}-\mathbf{u}_{w}\right) \cdot \mathbf{n}<0}\left|\left(\mathbf{e}_{i}-\mathbf{u}_{w}\right) \cdot \mathbf{n}\right| f_{i}(\mathbf{x}, t+\delta t)}{\sum_{\left(\mathbf{e}_{j}-\mathbf{u}_{w}\right) \cdot \mathbf{n}>0}\left|\left(\mathbf{e}_{j}-\mathbf{u}_{w}\right) \cdot \mathbf{n}\right| f_{j}^{e q}\left(\mathbf{x}, \rho_{w}, \mathbf{u}_{w}\right)} f_{k}^{e q}\left(\mathbf{x}, \rho_{w}, \mathbf{u}_{w}\right),
\end{aligned}
$$

where $\mathbf{u}_{w}$ and $\rho_{w}$ are the velocity and density at the wall, respectively, and $\mathbf{n}$ is the unit normal. The tangential momentum accommodation coefficient, $\alpha$, stands for the fraction of impinging molecules absorbed and re-emitted diffusely, while $(1-\alpha)$ are specularly reflected from the surface. The diffuse reflection at wall is assumed for $\alpha=1$.

Kramers' problem. - The model is initially tested on the Kramers' problem. The Kramers' problem consists of finding the molecular distribution function of a gas which fills the half-space $y>0$ bounded by a plate at $y=0$. There is a velocity gradient along the $y$ direction and this gradient becomes a constant as $y \rightarrow \infty$. In the simulation, the wall at $y=0$ is fixed and the Maxwellian kinetic boundary has been used with different tangential momentum accommodation coefficient $\alpha$. To achieve a constant shear rate, we apply a constant velocity $u_{w}$ for the upper wall at $y=L$ which is $200 \lambda_{0}$ away from the lower plate. In addition, we adopted an extrapolation scheme for non-equilibrium part to obtain the unknown distribution functions at the upper wall for non-slip flow [44]. Therefore, the upper wall has no influence on the Knudsen layer at the lower wall. To validate the present approach, our LB model results are compared with the data obtained by Loyalka et al [45] where the BGK equation is directly solved. Figure 4 shows that our LB model results agree well with the solutions of the BGK equation.

Planar Couette flow. - The second test case is a planar Couette flow consisting of a moving upper plate at $y=L$ and a stationary lower plate at $y=0$. The kinetic boundary condition given in Eq. (9) is employed to describe the molecular interactions with the solid walls, while periodic boundary conditions are implemented at the inlet and outlet. In Fig. 5, we demonstrate that modification on local mean free path enables our LB model to capture the nonlinear velocity profiles in the Knudsen layer. Without modification on local mean free path, Fig. 6 shows that the high-order D2Q16 LB model given by Ansumali et al. [31] still fails to describe the nonlinear velocity profiles in the Knudsen layer quantitatively. 


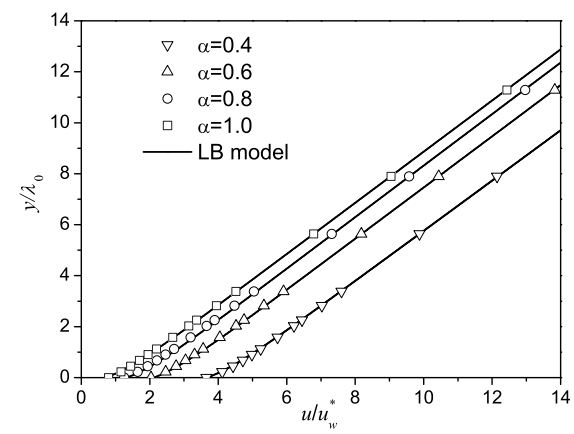

Fig. 4: The velocity profiles of the Kramers' problem with different tangential momentum accommodation coefficients. $u_{w}^{*}=(2 / \sqrt{\pi}) \lambda_{0} u_{w} / L$. The symbols are the data from Loyalka et al [45].

Therefore, appropriate wall boundary conditions or wall scaling treatment is also neccessary for high-order LB models.

Figure 7 illustrates the predicted velocity profiles for a planar Couette flow at $K=0.1-$ 1.0, mainly in the transitional flow regime. Our LB results are compared with the solutions of the linearised Boltzmann equation given by Sone et al. [46]. The predicted velocity profiles are in good agreement with the analytical solutions of the linearised Boltzmann equation, both in the bulk and wall regions. Particularly, we can see that the curvature near the walls region was captured well, which is a typical phenomenon of gas rarefaction effect, where the NS equations will predict a linear velocity profile. When the Knudsen number increases, the Knudsen layers start to interfere with each other so that the overlap effect of the Knudsen layers become significant for highly non-equilibrium flows. Figure 7 shows that our LB model can not only predict nonlinear velocity profile in the Knudsen layer but also the overlap effect of the Knudsen layers at the upper and lower walls.

Pressure-driven Poiseuille flow. - The third test case is fully-developed pressure driven Poiseuille flows with two parallel plates located at $y=0$ and $y=L$. We will compare our LB model results with the solution of linearised Boltzmann equation given by Ohwada et al. [47]. Ohwada et al. assumed the applied pressure gradient in the streamwise direction was small, so that the flow could be considered as incompressible. Therefore, a small uniform pressure gradient is applied in the streamwise direction in the current LB simulation. The periodic velocity boundary conditions are used again at the inlet and outlet.

Figure 8 illustrates that the predicted velocity profiles across the channel for $K=0.1-10$ where the velocity is normalized by the channel mean velocity $u_{m}$. The present LB results are in good agreement with the solutions of the linearised Boltzmann equation in both central and wall regions with $K$ as large as 4 . Therefore, the present LB model incorporating local mean free path can also capture the high-order rarefaction effect in pressure driven flows.

Summary. - A geometry-dependent local mean free path approach has been implemented in our simplified "high-order" LB model which enables our model to capture flow characteristics in the Knudsen layer. In addition, this approach has a distinguished advantage that is convenient to tackle complex geometries and interference of Knudsen layers.

$$
* * *
$$

The authors are grateful to the helpful discussion with I. Karlin, X. Shan, R. Barber and J. Reese. We also thank the editor S. Succi for his insightful suggestions. 


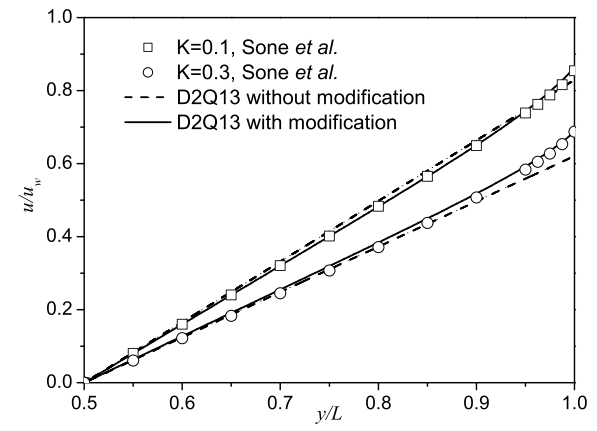

Fig. 5: The velocity profiles of the planar Couette flows. Our LB model results with and without local mean free path modification are compared with the solutions of the linearised Boltzmann equation given by Sone et al. [46].

\section{REFERENCES}

[1] Reese J. M., Gallis M. A. and Lockerby D. A., Philos. Trans. R. Soc. London, Ser. A, 361 (2003) 2967.

[2] Gad-el-HaK M., J. Fluids Eng., 121 (1991) 5.

[3] Lockerby D. A., Reese J. M. and Gallis M. A., Phys. Fluids, 17 (2005) 100609.

[4] Zhang Y. H., Qin R. S. and Emerson D. R., Phys. Rev. E, 71 (2005) 047702.

[5] Struchtrup H. and Torrilhon M., Phys. Fluids, 15 (2003) 2668.

[6] Gu X. J. and Emerson D. R., J. Comput. Phys., 225 (2007) 263.

[7] Xu K. and Li Z., J. Fluid Mech., 513 (2004) 87.

[8] Fan J. and Shen C., J. Comp. Phys., 167 (2001) 393.

[9] Baker L. L. and Hadjiconstantinou N. G., Phys. Fluids, 17 (2005) 051703.

[10] Chun J. and Koch D. L., Phys. Fluids, 17 (2005) 107107.

[11] Nie X. B., Doolen G. D. and Chen S., J. Stat. Phys., 107 (2002) 279.

[12] Lim C. Y., Shu C., Niu X. D. and Chew Y. T., Phys. Fluids, 14 (2002) 2299.

[13] Tang G. H., Tao W. Q. and He Y. L., Int. J. Mod. Phys. C, 15 (2004) 335.

[14] Tang G. H., Tao W. Q. and He Y. L., J. Appl. Phys., 97 (2005) 104918.

[15] Lee T. and Lin C. L., Phys. Rev. E, 71 (2005) 046706.

[16] Zhang Y. H., Qin R. S., Sun Y. H., Barber R. W. and Emerson D. R., J. Stat. Phys., $121(2005) 257$.

[17] Tang G. H., Tao W. Q. and He Y. L., Phys. Fluids, 17 (2005) 058101.

[18] Sbragaglia M. and Succi S., Phys. Fluids, 17 (2005) 093602.

[19] Toschi F. and Succi S., Europhys. Lett., 69 (2005) 549.

[20] Sofonea V. and Sekerka R. F., Phys. Rev. E, 71 (2005) 066709.

[21] Shan X. W., Yuan X. F. and Chen H., J. Fluid Mech., 550 (2006) 413.

[22] Zhang Y. H., Gu X. J., Barber R. W. and Emerson D R., Phys. Rev. E, 74 (2006) 046704.

[23] Guo Z. L., Zhao T. S. and Shi Y., J. Appl. Phys., 99 (2006) 074903.

[24] Zhang R., Shan X. and Chen H., Phys. Rev. E, 74 (2006) 046703.

[25] Sbragaglia M. and Succi S., Europhys. Lett., 73 (2006) 370.

[26] Zhang Y. H., Gu X. J., Barber R. W. and Emerson D R., EPL, 77 (2007) 3003.

[27] Niu X. D., Hyodo S. A., Munekata T. and Suga K., Phys. Rev. E, 76 (2007) 036711.

[28] Szalmás L., EPL, 80 (2007) 24003.

[29] Chikatamarla S. S. and Karlin I. V., Phys. Rev. Lett., 97 (2006) 190601.

[30] SucCi S., Phys. Rev. Lett., 89 (2002) 064502.

[31] Ansumali S., Karlin I. V., Arcidiacono S., Abbas A. and Prasianakis N. I., Phys. Rev. Lett., 98 (2007) 124502.

[32] He X., Luo L. S. and Dembo M., J. Comp. Phys., 129 (1996) 357.

[33] Tang G. H., Zhang Y. H. and Emerson D. R., Phys. Rev. E, 77 (2008) 046701 


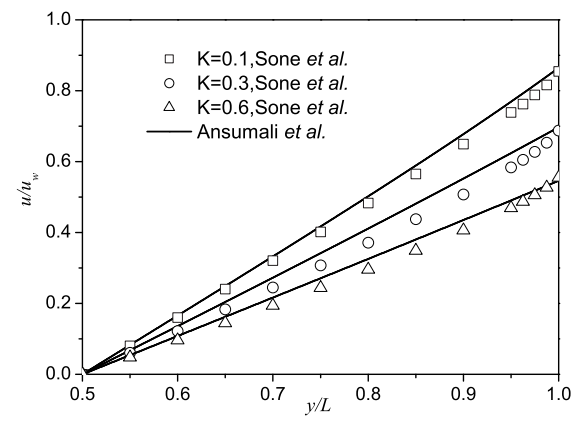

Fig. 6: The velocity profiles of the planar Couette flows. Our LB model results are compared with the analytical solutions given by Ansumali et al. [31]. The results of the linearised Boltzmann equation are given by Sone et al. [46].

[34] Karlin I. V. and Ansumali S., Phys. Rev. E, 76 (2007) 025701.

[35] Karlin I. V., Gorban A. N., Dukek G. and Nonnenmacher T. F., Phys. Rev. E, 57 (1998) 1668.

[36] Gorban A. N. and Karlin I. V., Physica A, 360 (2006) 325.

[37] Stops D. W., J. Phys. D, 3 (1970) 685.

[38] Guo Z. L., Shi B. C. and Zheng C. G., EPL, 80 (2007) 24001.

[39] Chen H., Kandasamy S., Orszag S., Shock R., Succi S. and Yakhot V., Science, 301 (2003) 633.

[40] Qian Y. H., D’Humières D. and Lallemand P., Europhys. Lett., 17 (1992) 479.

[41] Benzi R., Succi S. and Vergassola M., Phys. Rep., 222 (1992) 145.

[42] Chen S. and Doolen G. D., Annu. Rev. Fluid Mech., 30 (1998) 329.

[43] Ansumali S. and Karlin I. V., Phys. Rev. E., 66 (2002) 026311.

[44] Tang G. H., Tao W. Q. and He Y. L., Phys. Rev. E., 72 (2005) 016703.

[45] Loyalka S. K., Petrellis N. and Storvick T. S., Phys. Fluids, 18 (1975) 1094.

[46] Sone Y., Takata S. and Ohwada T., Eur. J. Mech. B-Fluids, 9 (1990) 273.

[47] Ohwada T., Sone Y. and Aoki K., Phys. Fluids A, 1 (1989) 1588. 

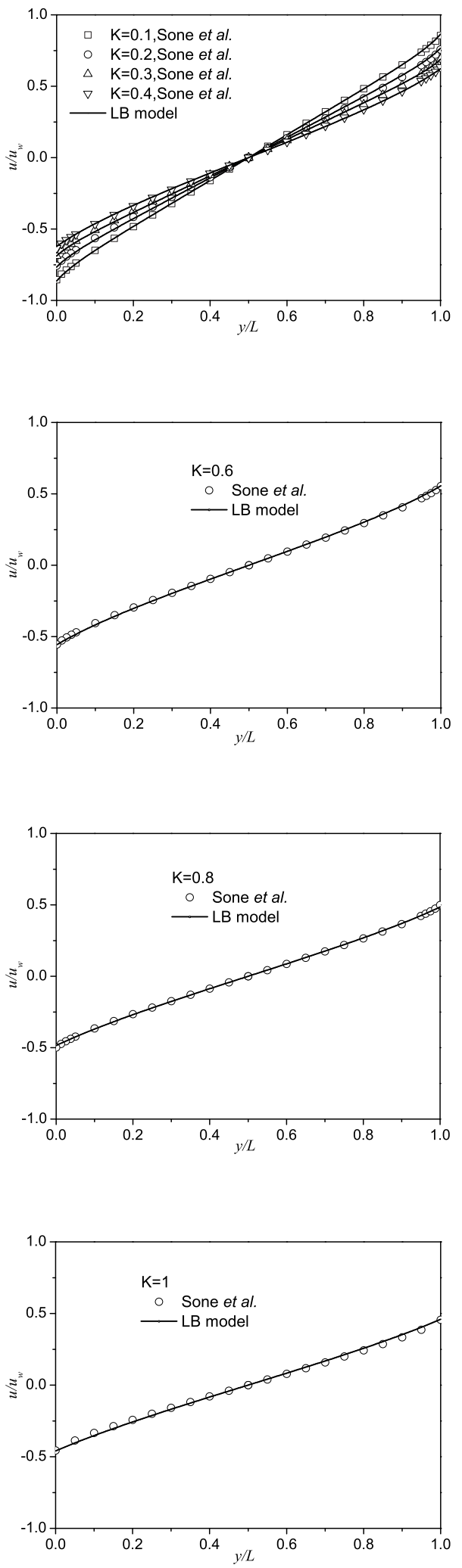

Fig. 7: The velocity profiles of the planar Couette flows. Our LB model results are compared with the solutions of the linearised Boltzmann equation given by Sone et al. [46].

$$
\text { p-9 }
$$



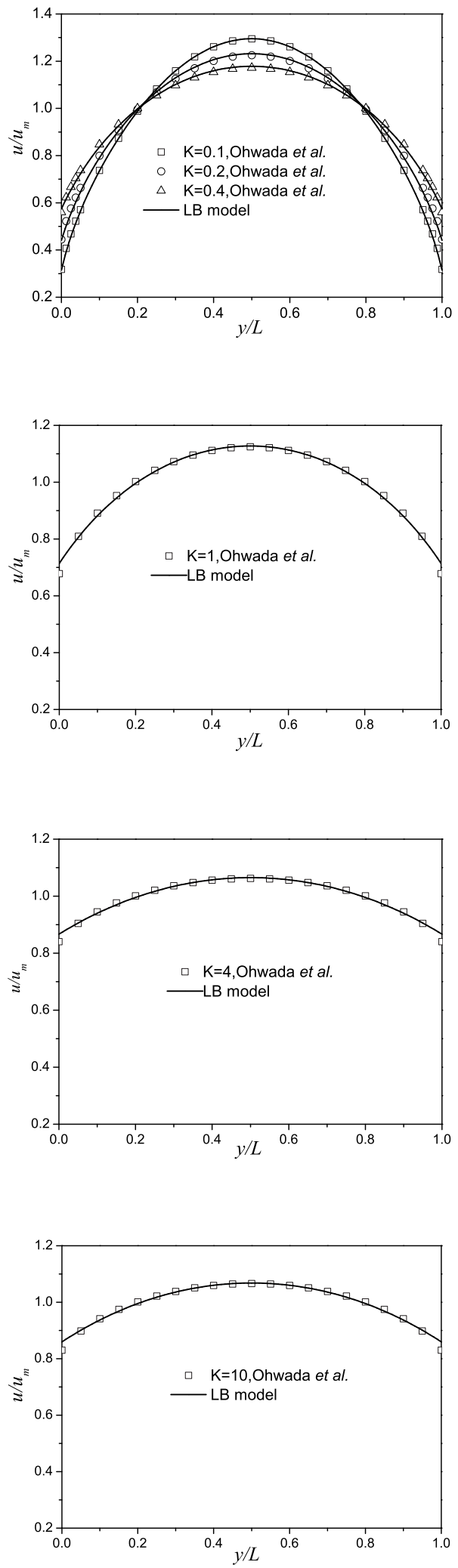

Fig. 8: The velocity profiles of the planar Poiseuille flows. Our LB model results are compared with the solutions of the linearised Boltzmann equation p-10 given by Ohwada et al. [47]. 